Psychotherapeut 2019.64:429

https://doi.org/10.1007/s00278-019-00385-x

(C) Springer Medizin Verlag GmbH, ein Teil von Springer Nature 2019

\section{Carsten Spitzer ${ }^{1}$ B Bernhard Strauß ${ }^{2}$}

${ }^{1}$ Klinik für Psychosomatik und Psychotherapeutische Medizin, Universitätsmedizin Rostock, Rostock, Deutschland

${ }^{2}$ Institut für Psychosoziale Medizin und Psychotherapie, Universitätsklinikum Jena, Jena, Deutschland

\title{
Narzissmus und Grenzverletzungen in der Psychotherapie
}

gesellschaftlichen und persönlichen $\mathrm{Zu}$ ständen hin.

Dies leitet über zu dem (oft narzisstisch motivierten) Machtmissbrauch in der Psychotherapie, der Gegenstand der folgenden drei, aufeinander bezogenen Beiträge ist. Sie stammen ausnahmslos aus der Kooperation des Ethikvereins e. V. (in Person von Andrea Schleu) und des Instituts für Psychosoziale Medizin des Universitätsklinikums Jena. Im ersten Beitrag beschäftigt sich Andrea Schleu (Essen) intensiv und systematisch mit dem Spektrum von Grenz- und Abstinenzverletzungen in der Psychotherapie. Es folgen die Ergebnisdarstellungen von zwei Master-Arbeiten, wobei zunächst Kontny et al. mithilfe eines inhaltsanalytischen Ansatzes die „Komplexität doppelter Beziehungsangebote“ in psychotherapeutischen Behandlungen beschreiben und Hannah Stuhler et al. abschließend auf die langsam eskalierenden Prozesse zunehmender Grenzverletzungen eingehen, die schlimmstenfalls zu sexuellem Missbrauch in einer Psychotherapie führen.

Trotz und gerade wegen aller Schwere und deprimierenden Grundtönung der Thematik von Narzissmus und Grenzverletzungen halten wir es für eminent wichtig, Psychotherapeutinnen und Psychotherapeuten für diese unterschätzte und sowohl wissenschaftlich wie klinisch unterrepräsentierte Problematik gleicher- maßen $\mathrm{zu}$ interessieren und zu sensibilisieren.

Wir wünschen uns, dass dieses Schwerpunktheft zumindest ein wenig dazu beitragen kann, und wünschen Ihnen eine interessante und hoffentlich für Ihre Arbeit gewinnbringende und fruchtbare Lektüre.

Carsten Spitzer (Rostock) und Bernhard Strauß (Jena)

\section{Korrespondenzadresse}

\section{Prof. Dr. Carsten Spitzer}

Klinik für Psychosomatik und Psychotherapeutische Medizin, Universitätsmedizin Rostock

Gehlsheimer Str. 20, 18147 Rostock, Deutschland

Carsten.Spitzer@med.uni-rostock.de

\section{Prof. Dr. Bernhard Strauß}

Institut für Psychosoziale Medizin und Psychotherapie, Universitätsklinikum Jena Stoystr. 3, 07740 Jena, Deutschland Bernhard.Strauss@med.uni-jena.de

Interessenkonflikt. C. Spitzer und B. Strauß geben an, dass kein Interessenkonflikt besteht. 